\title{
La transmisión del conocimiento entre los trabajadores de la parte operativa del distrito industrial
}

Este artículo presenta un estudio sobre la transmisión del conocimiento entre los trabajadores del distrito industrial en sus diversas formas: conocimiento tácito explícito y profesional, así como las características, fuentes, difusión, incorporación y generación del conocimiento en los distritos industriales. La metodología que se utilizó en esta investigación fue descriptiva con la recogida de datos a través de medios bibliográficos y documental. Este trabajo buscó contribuir con datos sobre la importancia de la transmisión del conocimiento para el distrito industrial y la necesidad de incentivos de ese proceso en el distrito industrial.

Palavras-chave: Conocimiento tácito; Conocimiento explícito; Distrito industrial; Transmisión de conocimiento.

\section{The transmission of knowledge among workers in the operational part of the industrial district}

\begin{abstract}
This article presents a study about the transmission of knowledge among workers of the industrial district in its various forms: tacit, explicit and professional knowledge, as well as the characteristics, sources, dissemination, incorporation and generation of knowledge in industrial districts. The methodology used in this research was descriptive with the collection of data through bibliographic and documentary means. This work sought to contribute with data about the importance of the transmission of knowledge to the industrial district and the need for incentives of this process in the industrial district.
\end{abstract}

Keywords: Tacit knowledge; Explicit knowledge; Industrial district; Transmission of knowledge.

Topic: Teoria Geral da Administração

Reviewed anonymously in the process of blind peer.
Received: 02/05/2018

Approved: 10/07/2018
Eduardo Dias Leite

Instituto Federal de Brasília, Brasil

http://lattes.cnpq.br/2110511717021627

eduardo.leite@ifb.edu.br

\section{Referencing this:}

LEITE, E. D.. La transmisión del conocimiento entre los trabajadores de la parte operativa del distrito industrial. Revista Brasileira de Administração Científica, v.9, n.2, p.1-12, 2018. DOI:

http://doi.org/10.6008/CBPC2179-684X.2018.002.0001
DOI: 10.6008/CBPC2179-684X.2018.002.0001 


\section{INTRODUCCION}

El conocimiento es entendido como la posesión de un conjunto de datos e información adquiridos por la educación o la experiencia, y que suponen la comprensión teórica o práctica de un tema; es la capacidad para convertir datos e información en acciones efectivas; o bien, atendiendo a enfoques más clásicos del pensamiento, combinados con posiciones religiosas, es el conjunto de creencias bien justificadas. Esas son definiciones genéricas, por lo que es conveniente buscar una mayor concreción a los efectos de acotar el tema dentro de la argumentación de ésta investigación que busca estudiar el conocimiento en los distritos industriales.

El conocimiento parece invisible, pero claramente conduce al desarrollo de las actividades laborales. El valor del conocimiento se incrementa cuando tiene un propósito clave y se enfoca en la misión, los valores fundamentales y las prioridades estratégicas. Los activos del conocimiento, como el dinero o el equipo, existen y merecen ser cultivados en el contexto de la estrategia utilizada para aplicarlos (WAH, 1999b; PASCARELLA, 1997; STEWART, 1995).

Consultando la base de datos de la plataforma Mendeley ${ }^{1}$, se encontró 3.597 publicaciones en revistas científicas, tesis doctorales, revistas profesionales, publicaciones periódicas, ponencias y actas y periódicos para los cinco años anteriores que hacían alusión al tema tratado en estas páginas. De ellas, la mayoría de las publicaciones pertenecían a revistas científicas, seguido de tesis doctorales. Se observó que no sólo no está aumentando el número de investigaciones sobre el tema, si no que se registró una ligera disminución en el año 2016, lo que lleva a deducir que el problema no está atrayendo el interés de los investigadores y que no hay demanda por parte del sector productivo con respecto a la transmisión de conocimientos entre los trabajadores, como presentamos en la tabla 1 y figura 1.

Tabla 1: Publicaciones sobre la transmisión de conocimientos entre los trabajadores.

\begin{tabular}{|c|c|c|c|c|c|c|}
\hline Año & Rev. científicas & Tesis doctorales & Revistas profesionales & Ponencias y actas & Periódicos & Total \\
\hline 2012 & 576 & 194 & 63 & 21 & 7 & 861 \\
\hline 2013 & 560 & 215 & 60 & 15 & 6 & 856 \\
\hline 2014 & 583 & 56 & 68 & 21 & 17 & 745 \\
\hline 2015 & 607 & 3 & 53 & 14 & 8 & 685 \\
\hline 2016 & 549 & 0 & 66 & 16 & 22 & 653 \\
\hline TOTAL & 2.875 & 468 & 310 & 87 & 60 & 3.797 \\
\hline
\end{tabular}

Apesar de un número expresivo de publicaciones sobre la transmisión de conocimiento, encontramos sólo 3 publicaciones que están más cerca del tema de esta investigación que es la transmisión de conocimientos entre los trabajadores del distrito industrial. El primer trabajo fue realizado en China por Li et al. (2015), que investigaron la difusión del conocimiento entre los trabajadores de la zona rural. El segundo fue realizado por Orduña (2013), que investigó la capacidad de innovación y la transmisión de conocimiento entre los investigadores y también entre los técnicos en el distrito industrial cerámico de Castellón y el tercero fue realizado por Martín-Cruz et al. (2009), que investigó la influencia de la motivación intrínseca y extrínseca sobre la transmisión de conocimiento en una organización sin fines de lucro, en

\footnotetext{
${ }^{1}$ Plataforma Mendeley, consultado en marzo de 2017.
} 
Valladolid, España.

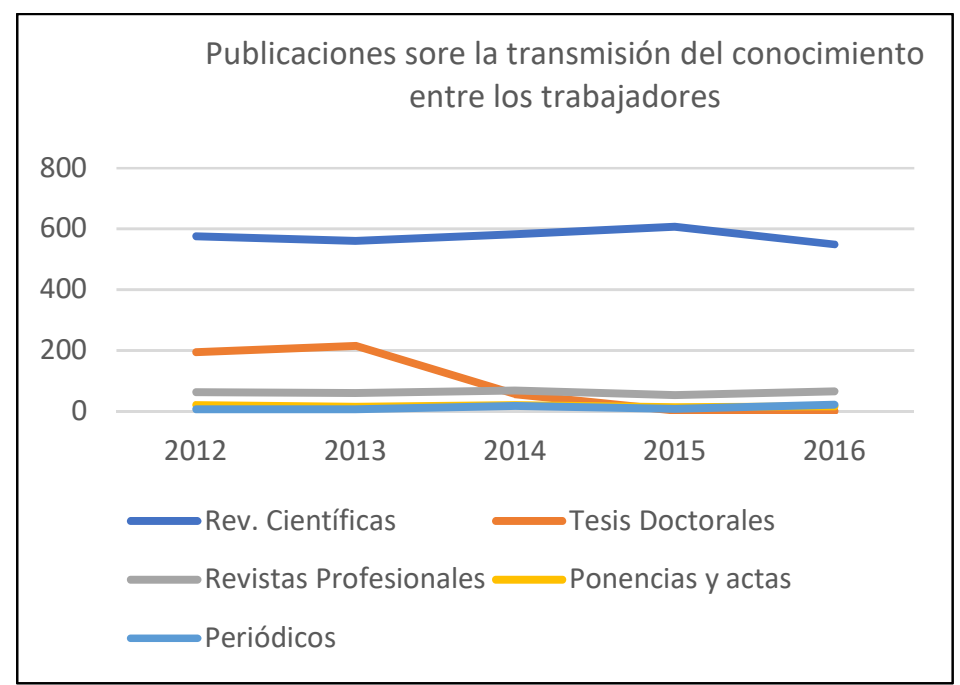

Figura 1: Evolución de las publicaciones sobre la transmisión de conocimientos.

La realización de este artículo buscó analizar la transmisión del conocimiento entre los trabajadores que se ocupan de la parte operativa de las industrias en el distrito industrial. Para hacer esta investigación, se trabajó con los tipos de conocimiento: explícito, que es el conocimiento que sabemos, tenemos y somos plenamente conscientes cuando lo ejecutamos; el conocimiento tácito, que es aquel que permanece en un nivel "inconsciente", se encuentra desarticulado y lo implementamos y ejecutamos de una manera mecánica sin darnos cuenta de su contenido.

El conocimiento profesional, que se refiere al conjunto de conocimientos y habilidades necesarias para funcionar con éxito en una profesión particular. El objetivo general de esta investigación es analizar la transmisión del conocimiento en los distritos industriales. La metodología utilizada en esta investigación fue descriptiva, con la recogida de datos a través de medios bibliográficos.

\section{DISCUSIÓN TEÓRICA}

\section{Conocimientos tácito y explícito}

Según Random House Dictionary of English Language, El conocimiento tácito es entendido sin ser expresado abiertamente, es el conocimiento para el cual no tenemos palabras. Para Liebowitz et al. (1998) el conocimiento tácito es automático, requiere escaso tiempo o pensamiento y ayuda a determinar cómo las organizaciones toman decisiones e influyen en el comportamiento colectivo de sus miembros.

El conocimiento tácito es altamente valorado en la tradición filosófica oriental. Así pues, mientras que el conocimiento explícito puede ser fácilmente procesado por un equipo informático, distribuido electrónicamente (por ejemplo, a través de correo electrónico) o almacenado en una base de datos, la naturaleza del conocimiento tácito dificulta enormemente su procesamiento y distribución. Para ello es necesario transformarlo en conceptos que todos podamos entender, es decir, es necesario convertirlo en conocimiento explícito (NONAKA et al., 1995). 
Nonaka et al. (2009) adopta una definición tradicional del conocimiento, como "creencia que es verdadera y justificada". Cabe recalcar que la epistemología occidental tradicional se ha centrado en la "veracidad" como el atributo esencial del conocimiento, destacando la naturaleza del conocimiento como "creencia justificada". Por lo tanto, mientras que la epistemología tradicional enfatiza lo absoluto, la vertiente estática y no-humana del conocimiento, típicamente expresado en proposiciones y lógica formal, considera el conocimiento como un proceso humano dinámico de justificación de creencias hacia la "verdad".

\section{La distinción entre conocimiento tácito y explícito}

El conocimiento tácito es difícil de expresar y verbalizar porque es expresado a través de habilidades basadas en acciones y no puede reducirse a reglas o recetas, está profundamente enraizado en la acción individual y en la experiencia, así como en ideales, valores o emociones que el sujeto adopte. Es un tipo de conocimiento altamente personal, difícil de formalizar, y por tanto difícil de comunicar y compartir con otros, así como de copiar.

Es aprendido a través de largos periodos de experiencia y de la realización de tareas, durante los cuales los individuos desarrollan una capacidad de hacer juicios intuitivos sobre el éxito en la ejecución de la actividad. Este conocimiento está profundamente arraigado en la acción, en el compromiso y en el desarrollo en un contexto específico. El dicho de que sabemos más de lo que podemos contar habla de la dimensión tácita del conocimiento (NONAKA et al., 1995; POLANYI, 1966; TEECE, 1998; JOSEFA et al., 2011).

Por su parte, el conocimiento explícito es aquel que se puede expresar en palabras y números, y es fácilmente comunicable y compartido. Es un tipo de conocimiento formal y sistemático, que puede basarse y explicitarse en objetos o reglas; de forma que el mero análisis y observación de dichos objetos y reglas nos permita conocer gran cantidad del conocimiento explícito existente en la organización.

El conocimiento explícito organizativo es aquel conocimiento residente en la organización, fruto de un proceso de aprendizaje organizativo o proceso de hacer explícito el conocimiento tácito que puede ser compartido por miembros de la organización y/o por agentes que interactúan con la organización, ya que se encuentra materializado en soportes de fácil acceso al estar expresado en documentos, bases de datos, informes, documentos, fórmulas científicas y/o procedimientos codificados las propias tecnologías, productos y/o servicios de la organización (NONAKA et al., 1995; POLANYI, 1966; TEECE, 1998; JOSEFA et al., 2011).

\section{Combinaciones del conocimiento}

Según Nonaka et al. (1995), existen cuatro tipos de combinaciones: socialización, combinación, articulación y internalización: Socialización (conocimiento armonizado) y Conocimiento tácito a conocimiento tácito; Combinación (conocimiento sistémico) y Conocimiento explícito a conocimiento explícito; Articulación o Exteriorización (conocimiento conceptual) y Conocimiento tácito a conocimiento explícito; y Internalización o Interiorización (conocimiento operacional) y Conocimiento explícito a conocimiento tácito. 
Socialización (conocimiento armonizado) y Conocimiento tácito a conocimiento tácito: En la socialización consiste en compartir experiencias. Se generan habilidades y conocimiento a partir de la observación, imitación y práctica. La parte fundamental para obtener conocimiento tácito es la experiencia. Es también compartir el conocimiento tácito a través de una comunicación cara a cara o de la experiencia compartida. Un ejemplo es el aprendizaje. La socialización tiene lugar cuando dos personas intercambian directamente conocimientos. Un ejemplo es la observación de la realización de un determinado trabajo por un colaborador. Este modelo básico consiste en una generación de conocimiento limitada, ya que este saber no se convierte en explícito, y por lo tanto no está a disposición de toda la organización. Por otro lado, esta transferencia también oferece una protección frente a las imitaciones;

Combinación (conocimiento sistémico) y Conocimiento explícito a conocimiento explícito: En la combinación las personas intercambian y combinan el conocimiento a través de distintos medios como por ejemplo: conversaciones por teléfono, documentos, etc. En esta parte se combinan o integran partes de conocimiento explícito y se sintetizan en un nuevo conocimiento. Combinación de varios elementos del conocimiento explícito: la construcción de un prototipo es un ejemplo. Los nuevos conocimientos explícitos se originan a través de la conjunción de otros saberes manifestados previamente. El conocimiento total de la organización no aumenta, ya que solo se resume o se expresa de otra manera lo que es conocido;

Articulación o Exteriorización (conocimiento conceptual) y Conocimiento tácito a conocimiento explícito: Se articulan los fundamentos del conocimiento tácito individual y se hacen intangibles y fáciles de compartir, para crear nuevo conocimiento. Nonaka et al. (1995) considera este proceso como clave en la creación del conocimiento. Desarrollando conceptos, que encajan con el conocimiento tácito combinado y que permiten su comunicación. En este modelo básico se consiguen conocimientos nuevos y valiosos para la organización y se ocupa una posición clave en el proceso de producción de habilidades en la organización, ya que transforma los conocimientos implícitos en las bases de la construcción de conocimientos explícitos. La explicación se produce a través del diálogo de los trabajadores, de la reflexión colectiva y de la concientización de los conocimientos; $y$

Internalización o Interiorización (conocimiento operacional) y Conocimiento explícito a conocimiento tácito: En la interiorización se presenta la conversión entre conocimiento explícito en conocimiento tácito, y esto está relacionado con el 'aprendiendo haciendo'. Se transmite el nuevo conocimiento explícito y quienes lo reciben lo internalizan para extenderlo, integrarlo y reestructurarlo en su conocimiento tácito. El saber explícito y documentado se interioriza en forma tácita por los trabajadores a través de la asimilación, de la complementación y de la reorganización de conocimientos. Este proceso está estrechamente relacionado con la creación de rutinas de acción o la adquisición de habilidades.

De los cuatro modos descritos, la conversión de conocimiento tácito en explícito es el mecanismo de transformación más rico en la creación de conocimiento, donde se liga el aprendizaje a la acción, el conocimiento explícito se convierte en parte de la base del conocimiento individual y se convierte en un activo para la organización. En mi medio de trabajo se aplica en gran medida el conocimiento explícito, porque se debe saber lo que se hace para realizarlo, aunque otros trabajos se realizan por simple intuición pero son muy pocos. En los estudios creo que se aplica una combinación de socialización, ya que se reciben 
conocimientos explícitos del profesor. Los conocimientos tácitos, aunque son muy útiles, son muy poco utilizados en las actividades que realizo.

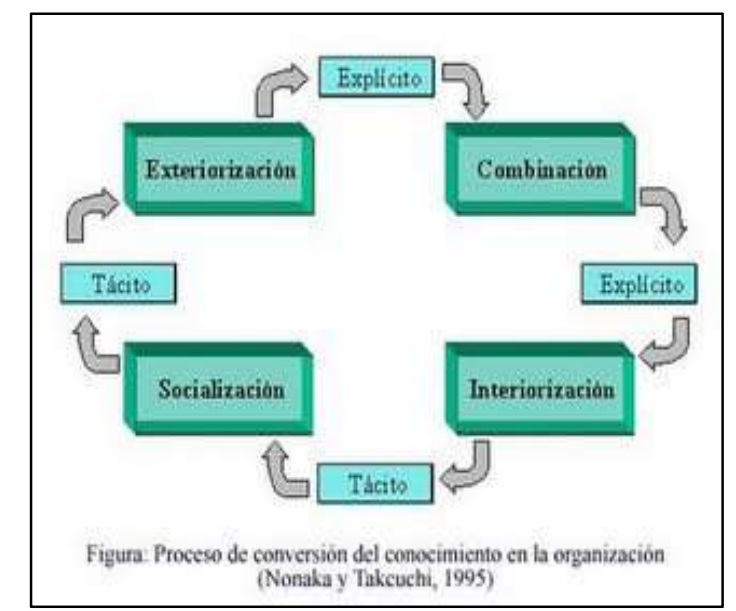

Figura 2: Proceso de conversión del conocimiento. Fuente: Nonaka et al. (1995).

\section{Conocimiento Profesional}

Por conocimiento profesional nosotros comúnmente nos referimos a ese cuerpo de conocimientos y habilidades que son necesarios para funcionar con éxito en una profesión particular. Este conocimiento está determinado por dos procedimientos comúnmente aceptados: análisis del trabajo o tarea; y consenso de la comunidad de personas que son reconocidos como profesionales en un campo particular (SHULMAN et al., 1986).

Mucho del conocimiento personal no está directamente relacionado con una profesión particular, tal como, por ejemplo, la enseñanza. Una parte de conocimiento existente en la estructura cognitiva de una persona se limita a afectar de algún modo su razonamiento, aplicación y adquisición de un conocimiento ulterior. Así, puede inferirse que hay al menos dos clases de relación entre conocimiento personal y profesional. El primero, y más obvio, es que una adquisición de conocimiento profesional dependerá de la estructura cognitiva existente en el trabajador individual y consecuentemente, como resultado de la interacción entre los dos, ese conocimiento construido será personal.

La segunda y más tácita clase de relación se refiere a la aplicación del conocimiento profesional. Mientras ciertos principios didácticos pueden ser transmitidos como los deseables por la dirección, por ejemplo, a través de un discurso, cada trabajador aplicará este discurso de forma claramente diferente, dependiendo de una gama completa de atributos personales incluyendo los heredados y los adquiridos (AUSUBEL, 1968).

\section{Conocimiento y sus características}

Algunas condiciones se exigen del conocimiento: capacidad de ser transmitido y, por lo tanto, entendido y asimilado por el receptor; reconocimiento y aprobación por parte de una comunidad experta; y capacidad para ser modificado y adaptado a nuevas situaciones. Esto es, supone que frente a situaciones concretas el individuo tiene la capacidad para pensar, interactuar y realizar acciones en consecuencia. El 
conocimiento como tal carece de valor cuando permanece estático y no es utilizado ni transmitido ni transformado. En este sentido adquiere importancia su difusión, en tanto que supone un ejercicio intelectual de comprensión y aprendizaje, así como los mecanismos a través de los cuales tiene lugar dicha transmisión de conocimiento y la velocidad a la que ésta tiene lugar.

Polanyi (1966) propone como punto de partida la necesidad de entender el conocimiento humano del hecho de que conocemos más de lo que podemos contar, estableciendo de estar forma la distinción entre conocimiento de tipo codificado y de tipo tácito. Se trata de aceptar la idea amplia del conocimiento y de que éste abarca mucho más de aquello que puede ser expresado en palabras o números, y que por lo tanto puede ser recogido en forma escrita o codificada (NONAKA, 1994).

Balconi et al. (2007) nos presentan tres tipos de conocimiento: Conocimiento como competencia para el desarrollo de ciertas atividades; Conocimiento como capacidad para conocer personas o cosas resultado de experiencia previa o familiaridade; y Conocimiento cuando se tiene certeza del mismo y por lo tanto se dispone de capacidad para interpretar y hacer uso de la información disponible.

La transmisión de conocimiento también ocurre por la relación dentro del distrito industrial. De acuerdo con Granovetter (1973), para entender la fuerza de los lazos, es necesario una combinación de factores tales como la cantidad de tiempo, la intimidad, la intensidad emocional, la confianza mutua y servicios mutuos que caracterizan el enlace.

En esta dimensión relacional, Molina-Morales et al. (2008) analizó la fuerza de los lazos (fuertes, frecuentes y interacciones íntimas) desde la perspectiva de los cuatro indicadores, a saber: de frecuencia, que identifica el número de veces que una persona (unidad) tuvo contacto con otra persona (unidad); la intimidad, que aborda el grado en que las relaciones son afectiva (por ejemplo, la amistad) y / o están basados en objetivos y propósitos comunes; las interacciones sociales; y finalmente, el grado en que los profesionales han trabajado en otras empresas en el mismo distrito de la región (red de empresas agrupadas territorialmente).

\section{Fuentes, difusión y generación del conocimiento}

En lo que se refiere a las fuentes del conocimiento se distingue entre internas y externas a las fronteras de la empresa. La distinción obedece al origen del proceso de captación del conocimiento e innovación, según tenga lugar en el interior de la empresa o sea adquirido a través de empresas o instituciones proveedoras de tecnología, innovación o conocimiento en sentido amplio. Se trata de una distinción en cuanto al origen y no tanto sobre la consideración de la innovación como endógena o exógena, que además de tener en cuenta el origen de los medios que facilitan el conocimiento tiene también en consideración la voluntad y planificación del proceso de innovación (BARNEY, 1995).

La localización geográfica favorece la pertenencia a un mismo colectivo sociocultural, por lo que en el caso de actividades productivas ubicadas en un mismo entorno físico, como es el caso de los distritos industriales, se dan los elementos idóneos para las relaciones cara a cara de los integrantes de la comunidad epistemológica así como para el establecimiento de contactos en su interior y, en concreto, para la movilidad 
de los trabajadores entre empresas pertenecientes a un mismo sector de actividad y que conforman una comunidad de conocimiento (ORDUÑA, 2013).

Según Muñoz (2006), los procesos de aprendizaje se van desarrollando alrededor de dos tipos de conocimiento: el tácito y el explícito. El conocimiento explícito es aquel que se puede expresar mediante palabras y números o cualquier otro dato codificado. Sin embargo, el conocimiento tácito, como la percepción subjetiva o las emociones, no se puede instrumentalizar y se transmite en determinados contextos y acciones; es muy personal y difícil de verbalizar o comunicar (POLANYI, 1966).

Es a través de los procesos de aprendizaje que la generación de conocimiento organizacional puede derivar en la creación de diferentes tipos de capacidades como: tecnológicas, aquellas que se refieren al complejo de habilidades, experiencias y esfuerzos conscientes que permiten a las empresas de un país comprar eficientemente, usar, adaptar, mejorar y crear tecnologías administrativas; aquellas que permiten desempeñar funciones que tienen que ver con decisiones orientadas a la institucionalización y al control empresarial; aquellas que tienen que ver con la toma de decisiones respecto a innovación, adaptación y cambio.

El proceso de aprendizaje que se desarrolla a través de dos tipos de conocimiento: el tácito y el explícito. Ambos tipos de conocimiento van interactuando en un proceso que describen en cuatro modelos básicos de producción y transferencia de conocimientos organizacionales. Uno de ellos es la socialización, la cual tiene lugar cuando dos personas intercambian directamente conocimientos. Un ejemplo es la observación de la realización de un determinado trabajo por un colaborador. Este modelo básico consiste en una generación de conocimiento limitada, ya que este saber no se convierte en explícito, y por lo tanto no está a disposición de toda la organización. Por otro lado, esta transferencia también ofrece una protección frente a imitaciones (NONAKA et al., 1995; CIPRÉS et al., 2005).

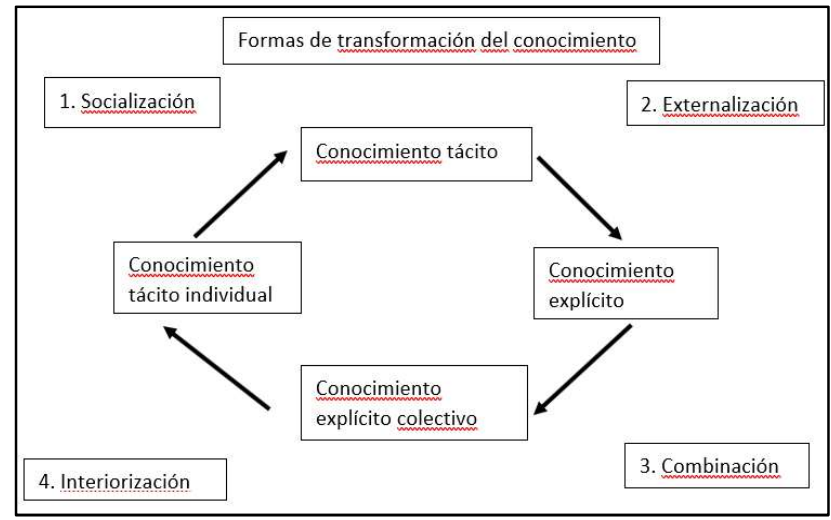

Figura 3: Formas de producción y transformación del conocimiento. Fuente: Nonaka et al. (1995).

Externalización: en este modelo básico se consiguen conocimientos nuevos y valiosos para la organización, ya que los conocimientos implícitos son documentados. Este modelo básico de exteriorización del conocimiento ocupa una posición clave en el proceso de producción de habilidades en la organización, ya que transforma los conocimientos implícitos en las bases de la construcción de conocimientos explícitos. La explicación se produce a través del diálogo de los trabajadores, de la reflexión colectiva y de la concientización de los conocimientos. 
Combinación: los nuevos conocimientos explícitos se originan a través de la conjunción de otros saberes manifestados previamente. El conocimiento total de la organización no aumenta, ya que solo se resume o se expresa de otra manera lo que es conocido.

Internalización: el saber explícito y documentado se interioriza en forma tácita por los trabajadores a través de la asimilación, de la complementación y de la reorganización de conocimientos. Este proceso está estrechamente relacionado con el "aprender haciendo", con la creación de rutinas de acción o la adquisición de habilidades.

Socialización: el conjunto de conocimientos implícitos/explícitos cuya transformación y transferencia de los individuos al grupo u organización se va dando a través de lo que Nonaka et al. (1995), llaman la "espiral de los conocimientos", que tiene en cuenta la creación del conocimiento organizativo como resultado de un proceso dinámico. El punto de partida del modelo son los trabajadores individualmente y su capacidad para crear y distribuir conocimientos entre los compañeros de trabajo, donde cada individuo valora la exteriorización de los saberes. Por otro lado, interioriza las experiencias del grupo completo.

\section{Distrito Industrial}

Para Becattini (1990), el Distrito Industrial se caracteriza por "la presencia activa de una comunidad de personas y una población de empresas en un área natural e históricamente limitada". El distrito industrial está comprendido por numerosas pequeñas empresas que desarrollan actividades relacionadas y que están localizadas en una comunidad claramente identificable. La homogeneidad cultural produce una atmósfera de cooperación y confianza donde la acción económica está regulada por normas implícitas y explícitas. En el distrito, a diferencia de otros entornos, la comunidad y las empresas tienden a fusionarse. Es esencial que cada empresa se especialice en una o unas pocas fases de los procesos de producción típicos del distrito (LAZERSON et al., 1999; GONCALO et al., 2012).

Para Haddad (1994), un distrito industrial es un conjunto de actividades que se desarrollan en un lugar determinado y pertenecen a un grupo o subsistema de actividades que están sujetas a las interrelaciones significativas de producción, la comercialización y la tecnología, donde las grandes empresas generan múltiples efectos de arrastre (efectos sobre la demanda local y efectos fiscales) en el desarrollo regional y local.

Para la existencia del distrito industrial, es necesario la interrelación de las pequeñas empresas con la población residente en este territorio, por lo que los aspectos socio-culturales de estas poblaciones son de suma importancia. Esta noción de arraigo es relevante porque la búsqueda de objetivos económicos siempre se acompaña de otros objetivos de carácter no económico, como la sociabilidad, la aprobación, la condición social o el poder. Por otro lado, la acción económica, ya que cada acción está socialmente "impregnada" y no se puede explicar por los movimientos individuales, ya que depende de las relaciones personales que tienen los individuos entre sí. Las instituciones económicas, como cualquier institución, no se determinan automáticamente por circunstancias externas, pero se construyen socialmente (BECATTINI, 1990; GRANNOVETTER, 1973). 
La existencia de economías locales externas a la empresa e interna al distrito industrial es uno de los elementos que justifican la importancia de la concentración geográfica entre las empresas. Por lo tanto, la presencia concentrada de empresas de la misma industria o segmento es capaz de generar algunos factores externos que sean apropiados para las empresas, con el aumento de su competitividad.

Las ventajas derivadas de la concentración geográfica se asocian no sólo al incremento del volumen de producción, sino también con la organización de las ganancias y desarrollo obtenidos a partir de una mayor integración entre los agentes. La concentración de productores especializados estimula la promoción de formas de integración entre los agentes, lo que hace que los secretos de la industria ya no sean secretos y vengan a "flotar en el aire", por lo que todo el mundo es capaz de absorberlos (MARSHALL, 1925; GONCALO et al., 2012).

Según Marshal (1920), la atmósfera del distrito industrial puede traducirse como la existencia de recursos intangibles basados en la experiencia, el conocimiento y en la información, que son comunes a las empresas del distrito y que se encuentran en el entramado socioeconómico del territorio donde se ubican las empresas y es posible la transmisión del conocimiento.

\section{METODOLOGIA}

La metodología utilizada en esta investigación fue descriptiva, con la recogida de datos a través de medios bibliográficos. Este trabajo buscó contribuir aportando referenciales teóricos sobre la importancia de la transmisión del conocimiento en el distrito industrial y sobre la necesidad de generar incentivos para fomentar este proceso en el distrito industrial.

Así, en cuanto a los aspectos metodológicos, la investigación tuvo la finalidad descriptiva, utilizándose medios bibliográficos para la recogida de datos. A forma básica de pesquisa teórica é a bibliográfica. La investigación en la literatura, según Vergara (2007), es el estudio sistemático desarrollado basado en el material publicado en libros, revistas, redes electrónicas, es decir, un material analítico para cualquier otro tipo de investigación que pueden ser la fuente primaria o secundaria.

Los datos, según Bardin (1977), se analizaron mediante análisis de contenido, que es "un conjunto de técnicas de análisis de comunicación que utiliza procedimientos sistemáticos y el contenido de la descripción de los objetivos de los mensajes", cuya intención es también para la inferencia de conocimientos relativos a las condiciones de producción (o, posiblemente, recibir), que utiliza indicadores (cuantitativos o no).

Para Bardin (1977), el "objetivo el análisis documental es la representación condensada de la información para la consulta y el almacenamiento; el análisis de contenido y el manejo de mensajes (contenido y la expresión de ese contenido) para resaltar los indicadores que se puede inferir sobre otra realidad que no el mensaje". 


\section{RESULTADOS Y DISCUSIÓN}

De acuerdo con el objetivo del trabajo, la investigación en la literatura es relevante en el momento en que busca comprender y discutir una teoría a través de una revisión de la literatura. Este trabajo tiene como objetivo insertar el tema de la transmisión del Conocimiento en el contexto de los trabajadores en el distrito industrial. Los resultados permiten concluir que tanto el proceso de generación de conocimiento como su difusión y transmisión al conjunto favorecen el desarrollo del distrito industrial. Nuestro trabajo pone también en evidencia la importancia que, para el distrito industrial, tienen los efectos de la creación de conocimiento y transmisión de información directa a los trabajadores de la producción a través de incentivos para la participación en conferencias, seminarios, proyectos y cursos de formación profesional.

Esta investigación pone también en evidencia la importancia que, para el DI, de los efectos de la transmisión del conocimiento entre los trabajadores y la vía de participación en proyectos de cursos de formación profesional. El proceso, lo que permitirá que las empresas en el distrito industrial puedan evaluar y mejorar este proceso.

\section{CONCLUSIONES}

En este artículo se pretendieron analizar las investigaciones llevadas a cabo sobre la transmisión de conocimientos entre los trabajadores en el distrito industrial, encontrándose que no hubo avances en las investigaciones en este campo de estudio e incluso hubo un ligero descenso en el número de publicaciones en el último año. La principal contribución de nuestra investigación son los resultados desde el punto de vista de los agentes involucrados en el proceso de transmisión del conocimiento de los trabajadores del distrito industrial. Una limitación de la investigación es la posibilidad de vistas parciales ofrecidas por la revisión de la literatura cualitativa, cuya única explicación es bibliográfica, sin los datos obtenidos a través de cuestionarios o entrevistas, no podía hacer explícita la transmisión del conocimiento entre los trabajadores del distrito industrial es decir, no explica con firmeza el fenómeno estudiado.

Como trabajo futuro, la limitación de esta investigación se puede considerar como un desafío a la continuidad de la investigación y, en consecuencia, se sugiere, por lo tanto, buscar el uso de métodos complementarios, como los métodos estadísticos se basan en correlaciones entre dos o más variables, y por lo tanto es posible establecer si la variable dependiente aumenta o disminuye en relación con la forma en que varía el valor de la variable independiente, y también el método de análisis de contenido por el análisis de entrevistas, para permitir la triangulación datos y para ampliar el poder de explicación y comprensión con respecto a los resultados de la investigación.

Por último, se señala que la investigación se centró en la transmisión del conocimiento en el contexto de los distritos industriales, un hecho que, aunque no es imposible generalizar los resultados, se puede complementar con otros análisis de la investigación en diferentes aglomeraciones territoriales y en la investigación en diferentes sectores. 


\section{REFERENCIAS}

AUSUBEL, D. P.. Educational psychology: A cognitive view. Cidade do México: Trillas, 1968.

BALCONI, M.; POZZALI, A.; VIALE, R.. The codification debate revisited: a conceptual framework to analyze the role of tacit knowledge in economics. Industrial and Corporate Change, v.16, n.5, p.823-849, 2007.

BARNEY, J. B.. Looking inside for competitive advantage. Academy of Management Executive, v.9, n.4, p.49-61, 1995.

BECATTINI, G.. The Marshallian Industrial District as a SocioEconomic Notion. In: PYKE, F.; BECATTINI, G.; SENGEBERGER, W.. Industrial Districts and Inter-firm Cooperation in Italy. Geneva: ILO, 1990. p.37-51.

CIPRÉS, M. S.; LLUSAR, J. C. B.. Concepto, tipos y dimensiones del conocimiento: configuración del conocimiento estratégico. Revista de Economía y Empresa, v.53, n.52, p.175-195, 2005.

GONÇALO, C. R.; REYES JUNIOR, E.. As redes sociais, uma ferramenta de diagnóstico para estratégias relacionais para a criação do conhecimento em arranjos produtivos locais (APL). Revista da Micro e Pequena Empresa, Campo Limpo Paulista, v.6, n.1, p.20-37, 2012.

GRANOVETTER, M.. The strength of weak ties. American Journal of Sociology, Chicago, v.78, n.6, p.1360-1380, 1973.

HADDAD, P. R.. Os Novos Pólos Regionais de Desenvolvimento no Brasil. In: VELLOSO, J. P. R.. Estabilidade e crescimento: Os desafios do Real. Rio de Janeiro: José Olympio, 1994.

JOSEFA, R. M.; INOCENCIA, M. L.; CANDELARIA, R. S.. Conocimiento Explícito en PYMEs. In: CONGRESO NACIONAL DE ACEDE, 11. Anais. Barcelona: 2011.

LAZERSON, M. H.; LORENZONI, G.. The Firms that Feed Industrial Districts: a Return to the Italian Source. Industrial and Corporate Change, v.8, n.2, p.235-266, 1999.

LEIBOWITZ, L.; BECKMAN, T.. Knowledge Organizations: What Every Manager Should Know. Boca Raton: CRC Press, 1998.

LI, Y.; SHI, D.; LI, X.; WANG, W.. Influencing factors of knowledge dissemination in rural areas in China. Nankai Business Review International, v.6, n.2, p.128-155, 2015.

MARSHALL, A.. Principles of economics. 8 ed. Londres: McMillan, 1920.

MARSHALL, A.. Principles of Economics. 8 ed. Londres: McMillan, 1925.

MARTÍN-CRUZ, N.; MARTÍN-PÉREZ, V.; TREVILLA-CANTERO, C.. Influencia de la motivación intrínseca y extrínseca sobre la transmisión de conocimiento: el caso de una organización sin fines de lucro. Revista de Economía Pública, Social y Cooperativa, v.66, p.187-211, 2009.

MOLINA-MORALES, F. X.. Los Distritos Industriales en la Europa Mediterránea: las diferencias entre Italia y España. Mediterraneo Económico, p.183-201, 2008.

MUÑOZ, G. V.. La generación de conocimiento organizacional como factor clave en el desarrollo de la capacidad de innovación: el caso de una empresa textil poblana. In: CONGRESO IBEROAMERICANO DE CIENCIA, TECNOLOGÍA, SOCIEDAD E IMNOVACIÓN. Anais. Cidade do México: 2006.

NONAKA, I.. A dynamic theory of organizational knowledge creation. Organization Science, v.5, n.1, p.14-37, 1994.

NONAKA, I.; KROGH, G. V.. Tacit knowledge and knowledge conversion: controversy and advancement in organizational knowledge Creation Theory. Organization Science, v.20, n.3, p.635-652, 2009

NONAKA, I.; TAKEUCHI, H.. The Knowledge Creating Company: How Japanese companies create the dynamics of innovation. Oxford: Oxford University Press, 1995.

ORDUÑA, V. B.. El papel moderador del capital social en los flujos de información y conocimiento en los Distritos Industriales. Tese (Doutorado) - Universitat Jaume I, Castellón de la Plana, 2013.

PASCARELLA, P.. Harnessing knOwledge, Management Review, p.37-40, 1997.

POLANYI, M.. The Tacit Dimension. New York: Doubleday, 1966.

SHULMAN, L. S.; SYKES, G.. A national board for teaching: in search for a bold standard: a report for the task force on teachinbg as a profession. New York: Carnegie Corporation, 1986.

STEWART, T. A.; KAUFMAN, D. C.. Getting real about brainpower. Washington: Fortune, 1995.

TEECE, D. J.. Competition, cooperation, and innovation: organizational arrangements for regimes of rapid technological progress. Journal of Economic Behavior \& Organization, v.18, n.1, p.1-25, 1992.

TEECE, D. J.. Research directions for knowledge management. California Management Review, v.40, n.3, p.289-292, 1998.

VERGARA, S. C.. Projetos e Relatórios de Pesquisa em Administração. 9 ed. São Paulo: Atlas, 2007.

WAH, L.. Making knOwledge stick. Management Review, p.24-29, 2007.

A CBPC - Companhia Brasileira de Produção Científica (CNPJ: 11.221.422/0001-03) detém os direitos materiais desta publicação. Os direitos referem-se à publicação do trabalho em qualquer parte do mundo, incluindo os direitos às renovações, expansões e disseminações da contribuiç̃o, bem como outros direitos subsidiários. Todos os trabalhos publicados eletronicamente poderão posteriormente ser publicados em coletâneas impressas sob coordenação da Sustenere Publishing, da Companhia Brasileira de Produção Científica e seus parceiros autorizados. Os (as) autores (as) posteriormente ser publicados em coletâneas impressas sob coordenação da Sustenere Publishing, da Companhia Brasileira de Produção Cientifica e seus parce 